\title{
Análisis econométrico sobre la generación y estructura del empleo a través de la demanda agregada y la inversión en Honduras, 2001-2011
}

Gabriel Perdomo ${ }^{1}$

\section{RESUMEN}

El objetivo de este artículo es analizar el fenómeno de la generación de empleo a través de las variables macroeconómicas (consumo privado, gasto público, inversión y exportaciones) en el sector laboral de la economía de Honduras, durante el periodo 2001-2011. Una vez caracterizado el sector laboral, el análisis se centrará en poder escenificar el efecto que tendrían en los niveles de empleo las principales medidas económicas de política pública, utilizando teorías de la economía moderna, específicamente modelos keynesianos y clásicos que se usan por su relación y acercamiento al comportamiento empírico de la economía de Honduras.

\section{METODOLOGÍA}

El análisis de esta información se efectuó a través de la relación de las variables macroeconómicas de demanda agregada e inversión que generan empleo en el sector formal de la economía hondureña, en donde se pretende relacionar el mercado laboral utilizando el número y las distintas desagregaciones del total de personas ocupadas, relacionándolo con el consumo público y privado con los problemas económicos laborales del subempleo visible e invisible, el desempleo abierto.

El proceso de correlación de las variables está centrado en poder comparar los efectos de las principales medidas de las políticas públicas en el marco de los planteamientos políticos de los principales candidatos a cargos públicos en las campañas electorales. El énfasis de este análisis estará centrado en poder

\footnotetext{
Profesor universitario beneficiario de una beca especial de la DICYP. Maestría en Metodologías de Investigación Económica y Social, Facultad de Ciencias Económicas, UNAH.
} 
proyectar posibles escenarios y efectos en los niveles de empleo.

Los resultados obtenidos de la presente investigación arrojan importantes fenómenos, en especial la propensión marginal a consumir de los hondureños, presentando un nivel alto de recurso destinado al consumo y el bajo nivel de ahorro, denotando que el consumo es el motor de mayor potencia en el crecimiento del PIB y la utilización de fuerza laboral que provienen de los sectores productores de bienes y servicios de consumo final, siendo estos el sector agropecuario y el comercio. Las recomendaciones están orientadas a estos hallazgos y muestran los retos de las políticas públicas en el marco político actual del año 2012 y 2013 . La función de esta investigación es poder proveer el comienzo de una aproximación para un debate técnico y amplio sobre la efectividad de las políticas públicas en materia de empleo, como una herramienta para la toma de decisiones que orienten tanto al sector privado como ente primario en la creación de puestos de trabajo, como para el sector público como ente creador de oportunidades y climas propicios para un empleo competitivo y digno para la población económicamente activa.

Palabras clave: PIB, propensión marginal a consumir, desempleo, impuestos, deuda pública, déficit presupuestario.

\section{ABSTRACT}

The aim of this paper is to analyze the phenomenon of generating employment through macroeconomic variables (private consumption, government spending, investment and exports) in the employment sector of the economy of Honduras, during the period 2001-2011. Where once characterized the labor sector this article will focus on to dramatize the effect they would have on employment levels of major economic policy measures using theories of modern economics, specifically classic Keynesian models and models that are used for its value and empirical approach to the behavior of the economy of Honduras.

The analysis of this information was made through the relationship of macroeconomic variables of aggregate investment, demand and employment generation in the formal sector of the Honduran economy, where we intend to link the labor market using the number and different breakdowns of the total of employed persons, relating to public and private consumption to labor economic problems visible and invisible underemployment, open unemployment. The correlation of variables is a process focused on to compare the effects of major public policy measures in the framework of policy approaches of the leading candidates for office 
in the election campaigns. The focus of this analysis will focus on possible scenarios to project and effects on employment levels.

The results of this research show significant phenomena especially the marginal propensity to consume of Hondurans presenting a high level of resources for consumption and low savings, implying that consumption is the most powerful engine of growth GDP and use of workforce coming from the sectors producing goods and services for final consumption being these agriculture and trade. The recommendations are intended has shown these findings and public policy challenges in the current political context of 2012 and 2013.

The function of this research is to provide an early approximation of a technical and broad debate on the effectiveness of public policies on employment, as a tool for decision -making to guide both the private sector as the primary entity in the creation of jobs and the public sector creates opportunities and supportive climates for being competitive and decent jobs for the economically active population.

Key words: GDP, marginal propensity to consume, unemployment, taxes, public debt, budget deficit. 


\section{INTRODUCCIÓN}

El fenómeno económico del empleo ha significado para los científicos, políticos y hacedores de políticas públicas un gran campo de estudio, ya que para la población hondureña este tema representa uno de los mayores intereses, por lo que demanda mayores esfuerzos y alternativas para hacer frente a los principales problemas de empleo: desempleo abierto, subempleo visible e invisible, en este último está representada una gran cantidad de hondureños de la población económicamente activa que se ubican en la condición de ocupados.

Desde el hito económico del ajuste estructural de la economía que se dio en un proceso generalizado en América Latina, se creó la expectativa de una intensa generación de empleo y una mayor equidad en el mercado laboral . Sin embargo, según un artículo de la CEPAL, Tendencias del empleo en los años noventa en América Latina, se concluye que los problemas laborales actuales se deben a la falta de crecimiento económico y al uso menos intensivo de mano de obra, causados principalmente por las transformaciones de las actividades productoras de bienes transables.

La modernización productiva de las empresas y la restructuración sectorial a favor de las actividades terciarias contribuyeron a la segmentación del mercado de trabajo sobre la base del nivel educativo, ya que la demanda laboral se inclinó a favor de la población que había cursado la enseñanza media y superior; en consecuencia, las personas con poca educación formal tuvieron un acceso menor al empleo asalariado y se amplió la diferencia de salarios entre los obreros calificados y no calificados. Al mismo tiempo se aumentó la diferencia de salarios entre las micro y medianas empresas, con las grandes empresas.

La restructuración sectorial del empleo contribuyó a desencadenar un proceso de heterogenización del mercado de trabajo que tiene diferentes dimensiones: generación simultánea e importante de empleo en los polos opuestos de la estructura laboral, mayor distancia entre lo formal y lo informal y más diferenciación entre los asalariados y los trabajadores por cuenta propia.

El caso de Honduras mantiene la misma tendencia que el promedio de Latinoamérica, ya que después del huracán Mitch se ensanchan estas características del sector laboral. En los países en desarrollo, por ejemplo, el desempleo suele ser reducido, lo cual no quiere decir que el mercado de trabajo sea 
muy eficaz, sino más bien que el desempleo constituye tan solo una parte del problema debidoa:

1. La falta de programas de asistencia a los desocupados: cuando un trabajador se encuentra sin empleo le resulta imposible permanecer sin trabajar, teniendo que dedicarse a cualquier actividad para sobrevivir, aun cuando esta no le ofrezca un empleo a tiempo completo en donde pueda sacar el suficiente partido a sus competencias u obtener ingresos que le permita llevar un modo de vida adecuado.

2. La alta proporción de trabajadores independientes: cuando estos trabajadores se enfrentan a períodos de baja actividad, prefieren dedicarse a actividades alternativas aunque les generan menos ingresos, en vez de quedar desocupados.

3. La existencia de modos tradicionales de organización del trabajo: las comunidades rurales suelen encargarse de absorber las tareas disponibles entre los miembros a costa de reducir las horas de trabajo y los ingresos de todos o de la mayor parte de los miembros de su población (Mata Greenwood, 1999).

Ante estas situaciones se analizarán los principales factores que confluyen en la generación de empleo y se orientará a los sectores más preponderantes en la utilización de mano de obra para poder absorber un mayor nivel de descopados y mejorar las remuneraciones de dichos sectores hacia el factor trabajo, para aumentar la demanda agregada y generar un ciclo de crecimiento económico.

\section{VARIABLES Y FUENTES DE INFORMACIÓN}

El desarrollo de esta investigación se realizó con base a la información de la encuesta permanente de hogares de propósitos múltiples del Instituto Nacional de Estadística de los años 2001 al 2011. Asimismo, se utilizó la base de datos referente a los indicadores macroeconómicos del Banco Central de Honduras en sus informes de la producción interna bruta por el enfoque del gasto.

El análisis de esta información se efectuó a través de la relación de las variables macroeconómicas de demanda agregada e inversión que generan empleo en el sector formal de la economía hondureña, en donde se pretende relacionar el 
mercado laboral utilizando el número y las distintas desagregaciones del total de personas ocupadas, relacionándolo con el consumo público y privado con los problemas económicos laborales del subempleo visible e invisible, el desempleo abierto. El proceso de correlación de las variables está centrado en poder comparar los efectos de las principales medidas de las políticas públicas en el marco de los planteamientos políticos de los principales candidatos a cargos públicos en las campañas electorales con vista a las elecciones internas 2012 y elecciones generales 2013. El énfasis de este análisis estará centrado en poder proyectar posibles escenarios y efectos en los niveles de empleo.

\section{BASE TEÓRICA}

Los enfoques teóricos están centrados en la macroeconomía moderna con los principales enunciados keynesianos y se complementan con teorías clásicas modernas. Entre estas se procederá en una secuencia lógica a un proceso descriptivo, la propensión marginal a consumir (PMC) de la economía hondureña, el modelo multiplicador del gasto y la demanda y oferta agregada. Después prosigue la investigación correlacional con los efectos de reducción de impuestos, aumento de gasto público, déficit presupuestarios y deuda pública, donde se utilizará como soporte teórico el enfoque ricardiano de la política fiscal.

\section{DISCUSIÓN}

\section{Etapa descriptiva}

En este apartado se expondrá la situación histórica de la evolución del fenómeno del empleo en Honduras, relacionado con el comportamiento desagregado de las principales variables del producto interno bruto (PIB) y del ingreso disponible que es el PIB menos todo recurso que sale al exterior, pago de depreciaciones del capital y pago de impuestos. El marco general del PIB se detalla en el gráfico 1. 


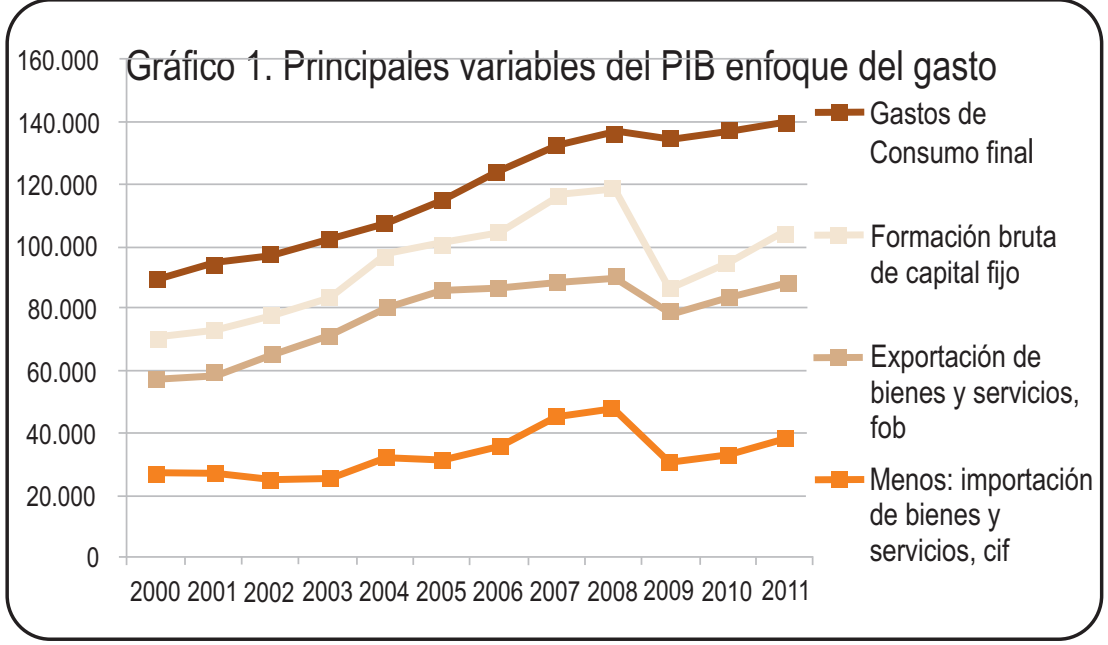

Fuente: PIB enfoque del gasto, Banco Central de Honduras.

En este gráfico 1 se puede observar la importancia del consumo privado y público en la generación del producto y como ente dinamizador de la economía, seguido por las exportaciones. Estas en el modelo keynesiano tienen un efecto negativo, ya que se destinan recursos para el exterior en su obtención. Es importante notar el bajo nivel de formación bruta de capital fijo que se da en el país, sugiriendo que la mejora de la inversión necesitaría de políticas públicas fuertes y de varios años para poder reactivar la inversión privada y pública en Honduras.

Las recomendaciones de este aspecto están orientadas a fortalecer la variable microeconómica principal, que es el consumo final de los hogares, empresas y gobierno, específicamente en los hogares en donde está directamente ligada con la generación de empleo y la pobreza, dotando de ingresos disponibles a sectores con bajo poder adquisitivo. Lo que respecta al ámbito del mercado laboral se explicará en los siguientes apartados.

\section{Contexto del mercado laboral en Honduras}

Los principales problemas a tratar en este segmento son:

1. Subempleados visibles: son las personas que en la semana de referencia trabajaron menos de 36 horas y en el momento de la entrevista expresaron deseo de haber podido trabajar más tiempo, indicando que no lo hicieron porque 
no encontraron más trabajo. Para medir el subempleo visible se toman tanto las horas semanales trabajadas en la ocupación principal como en la secundaria.

2. Subempleados invisibles: son las personas que habiendo trabajado 36 horas semanales o más, tuvieron ingresos mensuales inferiores al salario mínimo promedio mensual por rama de actividad económica y área geográfica. Para medir el subempleo invisible se toman los ingresos mensuales obtenidos en la ocupación principal y en la secundaria.

3. Desocupados: en esta categoría están agrupadas las personas afectadas por el desempleo abierto. Incluye a los cesantes (aquellas que tenían una ocupación, la perdieron por una causa cualquiera y durante la semana de referencia estuvieron activos buscando un empleo nuevo o tratando de establecer un negocio o finca propia) y a los trabajadores nuevos (que buscaron un empleo por primera vez).

Es importante notar que los datos provienen de la encuesta realizada para obtener datos a mayo de cada año, lo que muestra que este comportamiento está muy ligado al periodo anterior del mismo. Por ejemplo, la caída abrupta que se dio en mayo de 2003 de 143,943 personas menos ocupadas, se dio por el lento crecimiento del PIB real en el 2002 de 9.8 mil millones de lempiras; en cambio, en mayo de 2009 se registró el punto más alto de personas ocupadas, el cual creció de mayo de 2008 a mayo de 2009 en 234,488 ocupados, ya que el PIB real de 2008 aumentó en 28.9 mil millones de lempiras (ver gráfico 2).

Gráfico 2. Comportamiento de la población ocupada 2001-2010

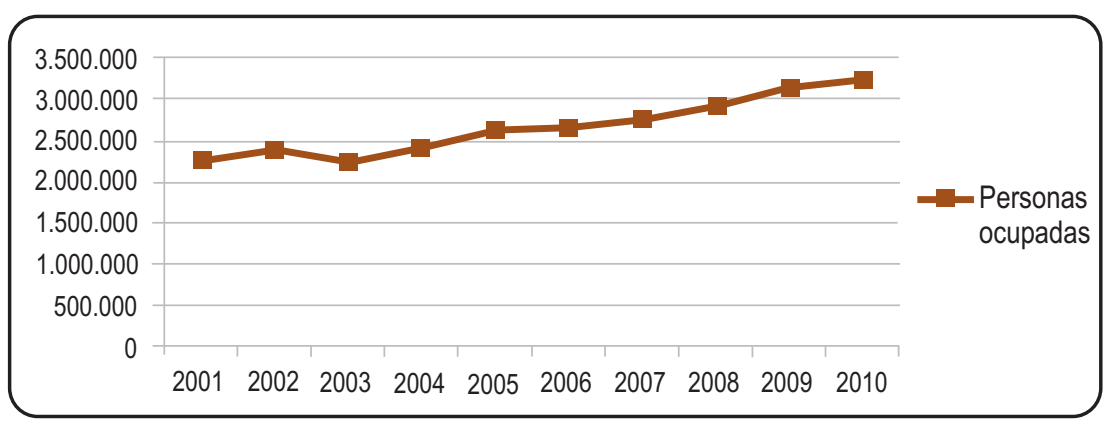

Fuente: EPHPM 2001-2010.

No se pueden apreciar abruptos cambios decrecientes, ni crecientes, en el comportamiento de las personas ocupadas, lo que está relacionado con el 
comportamiento del PIB que ha sido estable, pero con bajos niveles de crecimiento. En el gráfico 3 se muestra el comportamiento histórico de la última década de los tres fenómenos en cuestión, con sus respectivas tasas, donde el subempleo invisible es de mayor preponderancia, denotando que extensas proporciones de la población ocupada, es decir trabajando, recibe remuneraciones de bajo nivel a lo establecido en la legislación nacional con respecto al salario mínimo.

Gráfico 3. Problemas de empleo en Honduras

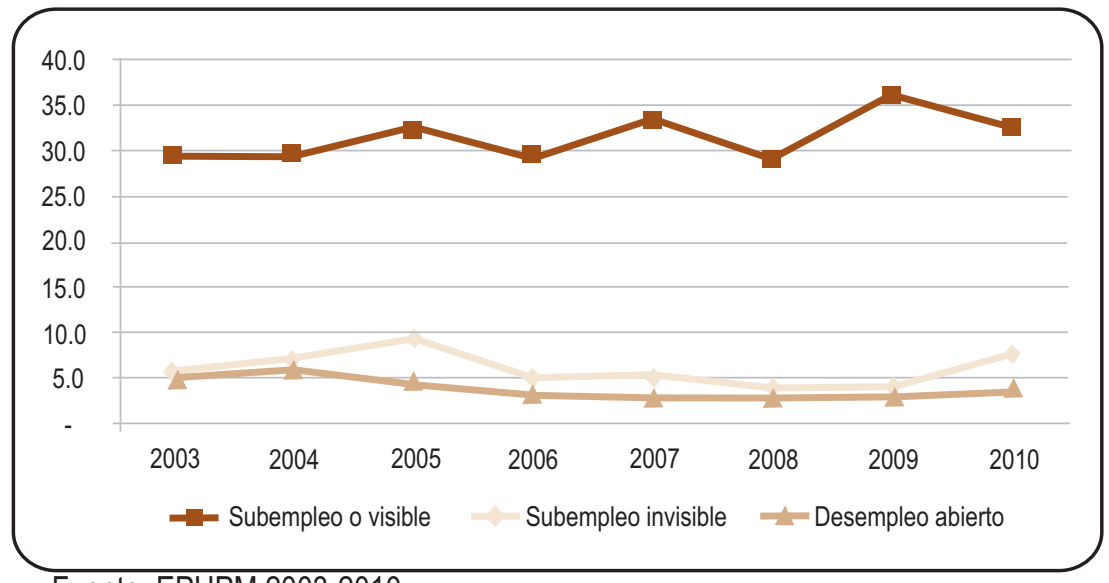

Fuente: EPHPM 2003-2010.

Se pueden apreciar las grandes cantidades de personas que están dentro de la población económica activa (PEA) que sufren de algún tipo de fenómeno negativo en el mercado laboral. Para el año 2010, se registró un total de 133,737 desocupados; 249,509 personas con subempleo visible y 1,061,702 personas con problemas de subempleo invisible, sumando $1,500,000$ personas con problemas de empleo. De una PEA de 3,387,717, los problemas representan un $45 \%$ del total de la PEA, dejando entrever el gran reto político y económico en el país en los siguientes años y dentro del marco de las elecciones internas 2012 y generales 2013, con una nueva estructura política de mayores opciones y diversidad ideológica, económica y social, lo cual hace que la generación de empleo sea el punto transversal de las propuestas políticas y centralizadas en los sectores económicos de mayor preponderancia en la utilización de mano de obra, tal como se muestra en el gráfico 4. 
Gráfico 4 . Principales sectores económicos que absorben la fuerza laboral

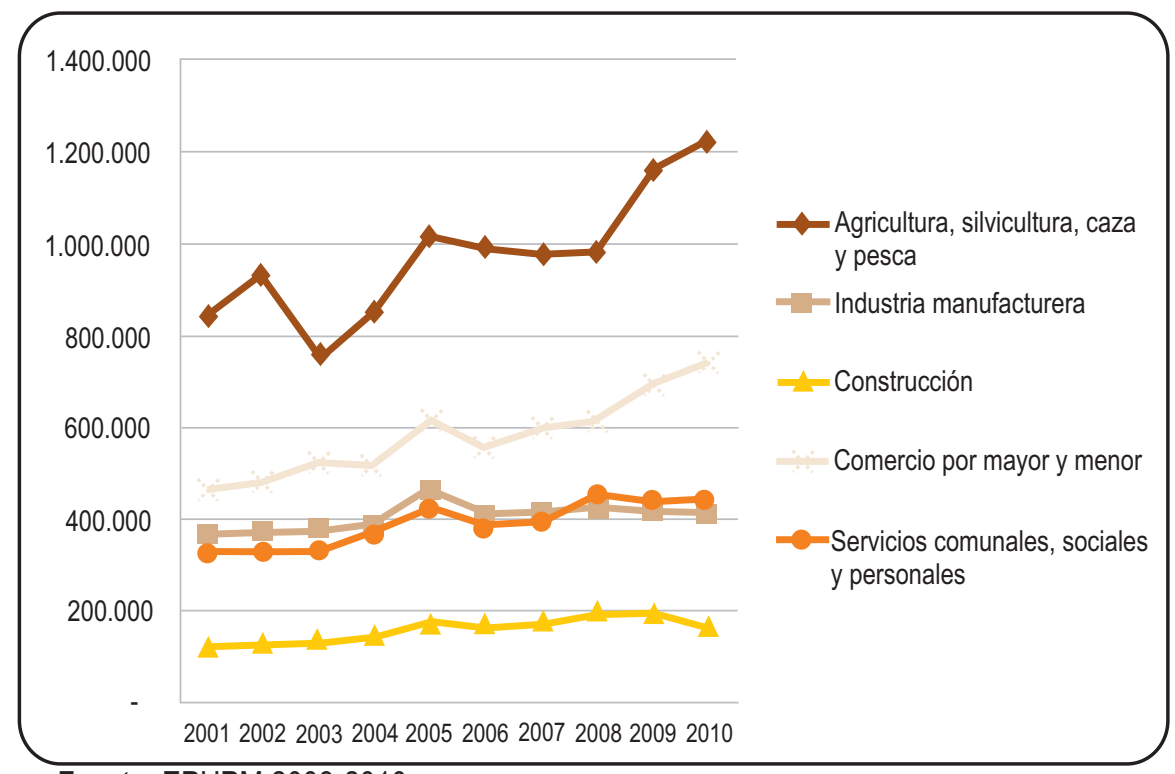

Fuente: EPHPM 2003-2010.

En los gráficos anteriores no se aprecia un comportamiento de ciclos económicos, ni abruptos cambios en los números de desempleados y subempleados, sino más bien un estancamiento laboral, esto se debe a la creciente utilización de capital en sustitución de la mano de obra y mayor especialización de la misma, que se complementa con el crecimiento lento, pero estable de la economía hondureña. Solamente presentan mayor dinamismo los sectores de agricultura, silvicultura, caza y pesca y el sector comercio, que son los que más personas absorben y ofertan al público bienes y servicios finales para el consumo.

Como se verá en los apartados siguientes, se comprueba la importancia del consumo en la economía y que un mayor nivel de gasto de los hogares específicamente dinamizaría los sectores que más empleos requieren, mejorando de esta manera los niveles de empleo y remuneración a la fuerza laboral. Por esta razón, una política pública, en especial la fiscal, debe ir orientada a mejorar el poder adquisitivo de los hogares para aumentar la demanda agregada, en donde crecería la necesidad de utilizar fuerza laboral para ampliar la oferta agregada de los principales productores de bienes y servicios que son bienes agropecuarios, específicamente alimentos para humanos y animales y bienes y servicios necesarios para la vida cotidiana, que son ofrecidos por el comercio y que este sector utiliza el factor humano en gran escala para su funcionamiento. 


\section{Modelos econométricos}

El siguiente modelo econométrico de una variable muestra la relación entre los valores absolutos del PIB real y valores absolutos de los ocupados, en donde estas variables tienen la relación directa positiva que se detalla a continuación, producto de lo cual se obtiene la ecuación siguiente:

\begin{tabular}{|c|c|c|}
\hline \multicolumn{3}{|c|}{ Estadísticas de la regresión } \\
\hline \multirow{2}{*}{\multicolumn{2}{|c|}{$\begin{array}{l}\text { Coeficiente de correlación múltiple } \\
\text { Coeficiente de determinación } \mathrm{R}^{\wedge} 2\end{array}$}} & 0.96755896 \\
\hline & & 0.93617035 \\
\hline \multicolumn{2}{|c|}{$\mathrm{R}^{\wedge} 2$ ajustado } & 0.92819164 \\
\hline \multicolumn{2}{|l|}{ Error típico } & 92468.0022 \\
\hline \multicolumn{2}{|l|}{ Observaciones } & 10 \\
\hline \multicolumn{2}{|c|}{ Análisis de varianza } & $\begin{array}{l}\text { Grados de } \\
\text { libertad }\end{array}$ \\
\hline \multicolumn{2}{|l|}{ Regresión } & 1 \\
\hline \multicolumn{2}{|l|}{ Residuos } & 8 \\
\hline \multicolumn{2}{|l|}{ Total } & 9 \\
\hline & Coeficientes & Error típico \\
\hline Intercepción & $1,611,984$ & 102,524 \\
\hline PIB real miles & 5,550 & 512 \\
\hline
\end{tabular}

Con un $80 \%$ de confianza en el modelo, se obtuvo que por cada mil millones de lempiras que crece la economía de forma real, es decir, deflactada de la inflación, el total de ocupados aumenta en 5,550; tal como lo muestra la pendiente del modelo. El intercepto, por su parte, denota que cuando la economía no registra un crecimiento en el periodo t, es decir en el año actual, la población ocupada se mantendría en 1 , 611,984 personas.

Las proyecciones del modelo presentan un reto para los hacedores de políticas públicas, ya que en este escenario para poder crear 100,000 personas empleadas el producto interno bruto real debería crecer en 15,000 millones de lempiras en un año, si se apunta a determinado objetivo. Es importante poder expresar que una cifra de gran magnitud como 15,000 millones equivalen al crecimiento del PIB real del año 2000 al 2004; dejando entrever la dificultad de que el alcance de un programa masivo pueda alcanzar más de cien mil puestos de trabajo en un año. Más adelante en este artículo se expondrán diferentes alternativas y políticas públicas orientadas 
al crecimiento económico como sustento para la creación de empleos. En el gráfico 5 se muestra la magnitud de un crecimiento de 15,000 millones, en el cual, en promedio, Honduras crece entre 5 a 8 mil millones de lempiras por año en el PIB real.

Gráfico 5. Comportamiento del PIB real 2000-2011

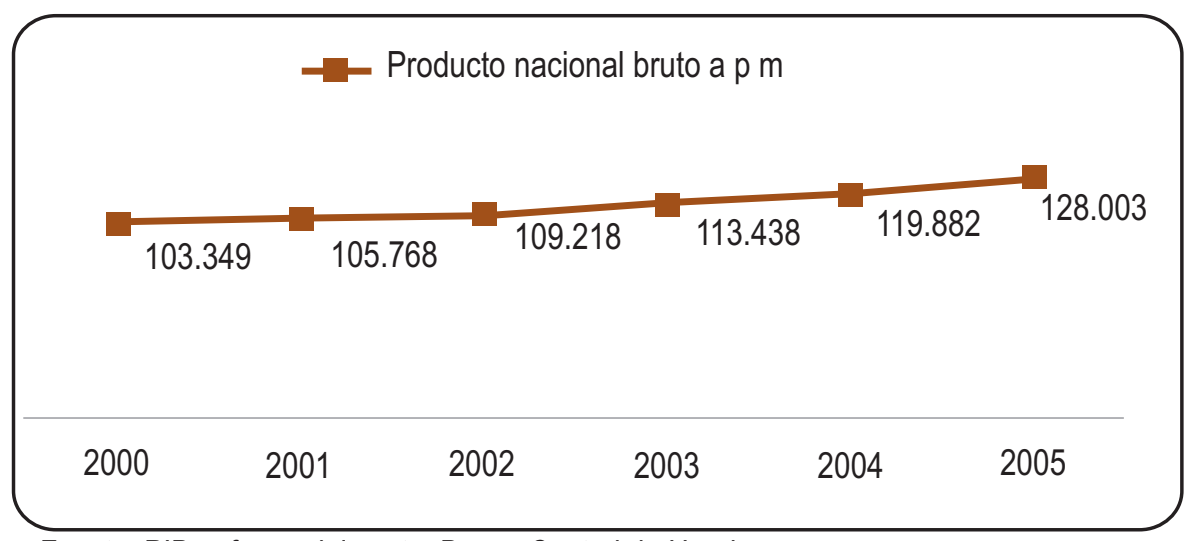

Fuente: PIB enfoque del gasto, Banco Central de Honduras.

Ante el contexto económico que se ha mantenido en la última década en Honduras, se visualizan varias tendencias a nivel macroeconómico, tales como el lento avance en el aumento de la producción y, consecuentemente, se ve debilitado el ingreso disponible de los hogares y las empresas necesario para el consumo y ahorro.

Para efecto de la modelización de la economía de Honduras de una forma simple y veraz, se ha seleccionado el modelo del multiplicador del gasto, que consiste en la premisa de que un aumento en los gastos exógenos (consumo privado, inversión, gasto gubernamental o exportaciones) de una unidad monetaria, el PIB aumenta en un cambio mayor a la unidad del gasto exógeno. Es decir, que si aumenta el gasto en 1 lempira, el PIB aumenta en más de un lempira por la sucesión de la utilización del recurso; en otras palabras, si se construye una casa se le paga L.100 al albañil, este utiliza L.80.00 y compra comida, el dueño del restaurante utiliza L.60 y compra los víveres generando una cadena de aumento de la riqueza y es a este proceso que la política pública debe estar orientado, a cómo poder crear mayor poder adquisitivo que genere a su vez riqueza en los diferentes agentes económicos de Honduras.

El modelo del multiplicador del gasto presenta varios supuestos o hipótesis en donde los salarios y precios permanecen fijos y hay recursos (tierra, trabajo, capital) 
sin emplear. Además, se suprime el papel de la política monetaria y no hay reacciones de los mercados financieros ante cambios en la economía.

A nivel teórico, los economistas con base en la evidencia estadística y comportamiento del mercado real y financiero de Estados Unidos catalogan como el modelo del multiplicador como simple, pero en el contexto hondureño con mecanismo legales para fijar salarios y precios, mercados financieros no desarrollados y una política monetaria estable y constante en el tiempo, el modelo se ajusta de forma representativa a la evidencia empírica de Honduras, en donde existen recursos no utilizados.

El elemento más importante del modelo del multiplicador es la propensión marginal a consumir PMC, que consiste en la cantidad adicional que las personas consumen cuando reciben un lempira adicional de ingreso disponible (Paul Samuelson, 2005).

La pendiente de un modelo econométrico representa la PMC, en este caso, en la función de consumo de la economía hondureña proyectándola con un $80 \%$ de confianza y un grado de determinación del $98 \%$; se obtuvo el resultado siguiente:

$$
\text { Multiplicador del gasto }=\frac{1}{1-\text { PMC }}
$$

Se obtiene un multiplicador del gasto del 6.48, lo que significa que por cada lempira que se gaste en sueldos y salarios de los burócratas, construcción de carreteras, servicios de internet, salidas al cine, almuerzos en restaurantes, etc., el PIB aumenta en 6.48 lempiras. Este alto indicador es provocado por la alta propensión a consumir de los hondureños.

Ante esta situación, qué camino deben tomar los hacedores de políticas públicas para aumentar el PIB y para que el número de ocupados crezca en la economía. En la teoría keynesiana el Estado a través de su gasto e inversión pública es un ente dinamizador de la economía, de modo que se presenta la gran disyuntiva de reducir impuestos para aumentar el ingreso disponible de los hogares y las empresas y aprovechar el alto nivel de consumo para aumentar el PIB o acelerar el gasto gubernamental manteniendo constante los impuestos para crecer en una magnitud de 6.48 por cada lempira gastado. Ante estas inquietudes económicas se expondrán las ventajas y desventajas de la reducción de impuestos y aumento del gasto y se introducirán los fenómenos económicos del enfoque ricardiano de la política fiscal, el déficit presupuestario y deuda pública; elementos claves para una efectiva política económica de generación de empleo. 
La política fiscal y el desempleo: la reducción de impuestos como medida de política fiscal

Una reducción de impuestos en un periodo de depresión estimularía el gasto dejando mayor cantidad de dinero en manos de los individuos y el público para emplearlo en consumo e inversión (Due,1968). Utilizando este nuevo ingreso en bienes y servicios provenientes del sector agropecuario y comercial, en menor porcentaje se utiliza en el sector construcción, ya que los dos primeros sectores tienen mayor dinamismo en la generación de empleo por sus respectivas demandas agregadas que presionan para un aumento de la oferta agregada.

Es importante notar que el tema de precios no se ha tomado de forma preponderante en este artículo, ya que la inflación en Honduras es producida en gran medida por aumentos de costos de producción provenientes de los combustibles e impuestos y no por fuerzas de la oferta y demanda. Por ende, un aumento en el poder de compra no afectaría en gran medida el nivel de precios, si este aumento proviene de la reducción de impuestos regresivos como los tributos al combustible y demás.

En momentos de recesión no es necesario restringir el consumo privado mediante los impuestos, ya que el objetivo es utilizar los factores productivos ociosos en la economía aprovechando la alta propensión marginal a consumir, un aumento en el ingreso disponible mediante reducción de impuestos aumentaría el consumo e inversión privada, demandando mayores niveles de mano de obra y de capital.

En este esquema entra en acción la estructura tributaria regresiva en la cual Honduras está sumida y un programa de reducción de impuestos regresivos, como los impuestos al combustible, sobre ventas y actividades específicas aumentarían el poder adquisitivo de sectores de bajos ingresos que fortalecería la demanda agregada de bienes y servicios. Es muy importante mantener impuestos a actividades y bienes nocivos como el alcohol y el tabaco, ya que el consumo de estos impactan en la depreciación y capacidad de la mano de obra nacional y sus externalidades negativas presionan los servicios de salud, aumentando el gasto en los mismos.

Las ventajas de una reducción de impuestos son la rapidez de implementación, ya que estos programas no requieren de planes operativos, contratación de personal, licitaciones y procesos de adjudicaciones como lo presenta la inversión pública, solamente se requiere la puesta en marcha por el Congreso Nacional. La reducción de costos provenientes de los impuestos produce una creciente producción de 
bienes y servicios que los hogares y las empresas que desean, requieren y aspiran a obtener.

Las limitaciones a un programa de reducción de impuestos sería que una disminución en impuestos sobre la renta traerían beneficios a sectores que poseen actualmente un nivel de ingreso alto y se dejaría por fuera un aumento del poder adquisitivo a los sectores de bajos ingresos. En la eventualidad de una reducción de impuestos, la evidencia empírica de los comportamientos microeconómicos de los hogares hondureños sugiere que el efecto del enfoque ricardiano de la política fiscal sería muy propenso. Este enfoque, que es una crítica a la economía keynesiana, indica sobre si los hogares sabrán que si el gobierno disminuye los impuestos, pero no modifica los gastos, este incurrirá en un déficit presupuestario e incurrirá en obtención de préstamos, aumentando la deuda pública; entonces, si mantiene los gastos inevitablemente en el futuro el gobierno tendrá que elevar los impuestos para pagar los intereses de los préstamos. Según el enfoque ricardiano los consumidores que tienen expectativas racionales incrementarán sus ahorros y no el consumo, permaneciendo estable sin cambios en la producción interna bruta.

Ante esta situación se requiere de un gobierno que busque un presupuesto equilibrado y controle la deuda pública, lo que lleva a los siguientes puntos:

1. Incremento en el déficit del gasto público: un programa de reducción de impuestos y gastos gubernamentales llevaría a un inevitable déficit en el presupuesto de forma inmediata, ya que menores recaudaciones impactarían en el equilibrio presupuestario. Pero este déficit no sería tan grande, ya que un mayor nivel de ingreso aumentaría el consumo y como esta actividad está cargada con tributos cierta porción del sacrificio fiscal retornaría a las arcas del Estado, ya que la estructura tributaria regresiva del país obtiene sus recursos de los impuestos indirectos al consumo y venta de bienes y servicios. Aunque siempre existiría un déficit vendría a colación el siguiente fenómeno económico que es de suma importancia manejar de forma eficiente en su comportamiento: la deuda pública.

2. El crecimiento de la deuda pública: el financiamiento de las deudas públicas en Honduras son un campo de debate, ya que teóricos keynesianos abogan por déficits en el presupuesto para fomentar el gasto y consumo, lo cual a corto plazo es beneficioso, pero cuando se llega a niveles en donde se utilizan 14,000 millones de lempiras en concepto de servicio de la deuda, suenan las alarmas y ponen en duda la efectividad del endeudamiento. La cantidad que se utiliza para 
pagar intereses y capital de la deuda pública es mayor que los 10,000 millones de lempiras que la Secretaría de Salud, presupuesto en el año 2012, usó para producir servicios de salud a la población. Las grandes cantidades de recursos para el pago del servicio de la deuda significan una gran carga para la economía hondureña, ya que estos se financian vía tesoro nacional. Para una política fiscal efectiva de reducción de impuestos se debe mantener el gasto del gobierno y racionalizar el endeudamiento público. Ante las situaciones y fenómenos económicos descritos y correlacionados en los capítulos anteriores, se procederá a las conclusiones y recomendaciones para poder elevar y mejorar los niveles de empleo.

\section{CONCLUSIONES YRECOMENDACIONES}

En consonancia con el objetivo del presente artículo de poder analizar el fenómeno de la generación de empleo, se utilizarán los resultados de este artículo para poder recomendar acciones concretas de políticas públicas encaminadas al sector laboral. Los resultados del análisis son los siguientes:

1. El principal problema que afronta la fuerza de trabajo es el subempleo invisible, en donde los ocupados trabajan las horas estipuladas, pero reciben remuneración por su trabajo menor que lo establecido, socavando el poder adquisitivo de los hogares.

2. Los principales sectores que absorben la masa laboral son el sector agrícola, pecuario y comercio, con la mayoría de los ocupados empleados en estos sectores.

3. Los hogares hondureños destinan 85 centavos por cada lempira de ingreso a financiar el consumo de bienes y servicios finales. Denotando el bajo nivel de ahorro de las familias

4. Una reducción de impuestos regresivos aumentaría el ingreso disponible, aumentando la demanda agregada y fortaleciendo el crecimiento del producto interno bruto que utilizaría mayores niveles de empleo. 
Recomendaciones de políticas públicas

1. Acorto plazo

a. Programa de reducción de impuestos regresivos, especialmente los impuestos al combustible para mejorar el ingreso disponible.

b. Mantener el nivel actual, mejorar la eficiencia y transparencia de las asistencias sociales que propicien el poder de compra de familias de bajos ingresos.

c. Ampliar los beneficios fiscales a pequeñas y medianas empresas.

d. Liberar los procesos de puesta en marcha de negocios y empresas.

e. Control del gasto público.

f. Uso racional del endeudamiento público.

g. Fortalecimiento de los impuestos directos y recaudación más eficiente de los impuestos indirectos.

h. Aumentar los impuestos a bienes y actividades nocivas como el alcohol y tabaco para que el ingreso disponible no sea absorbido por el consumo de estos bienes que presentan externalidades negativas.

2. Alargo plazo

a. Inversión en infraestructura para favorecer la producción agropecuaria y el comercio.

b. Mejoramiento de la estructura de generación de energía eléctrica para controlar que los precios por energía no aumenten los costos de producción.

c. Utilización de la política monetaria para control de precios de bienes y servicios y control del tipo de cambio. 


\section{BIBLIOGRAFÍA}

Due, J. F. (1968). Análisis económico de los impuestos. Buenos Aires: Eudeba.

Instituto Nacional de Estadística. (2011). Encuesta permanente de hogares 2011. Tegucigalpa.

Keynes, J. M. (1936). Teoría general del empleo, el interés y el dinero. Madrid: Fondo de Cultura Económica de España.

Mata Greenwood, A. (1999). Definiciones internacionales y futuras del subempleo. Ginebra: Oficina Internacional del Trabajo.

Paul Samuelson, W. N. (2005). Economía. México DF: Mc Graw Hill Interamericana.

Roberto Sampieri, C. C. (2006). Metodología de la Investigación. México D.F: Mc Graw Hill.

Weller, J. (2000). Tendencias del empleo en los años noventa en América Latina y el Caribe. Revista de la CEPAL, 72. 